\title{
TAXONOMIC UPDATE OF THE SABELLIDS (POLYCHAETA: SABELLIDAE) FROM CHILE AND TAXA ESTABLISHED BY PROF. ERNST EHLERS, WITH A KEY TO GENERA OF SABELLINAE.
}

\author{
ACTUALIZACIÓN TAXONÓMICA DE LOS SABÉLIDOS (POLYCHAETA: \\ SABELLIDAE) DE CHILE Y TAXA ESTABLECIDOS POR EL PROF. ERNST \\ EHLERS, CON UNA CLAVE PARA LOS GÉNEROS DE SABELLINAE.
}

María Ana Tovar-Hernández ${ }^{1}$

\begin{abstract}
RESUMEN
El listado más reciente de poliquetos de Chile fue publicado en 1985. Desde entonces, se han efectuado una serie de contribuciones sistemáticas de la familia Sabellidae, lo que ha ocasionado cambios en el estado taxonómico de varios géneros y especies. Este trabajo proporciona nuevos nombres, sinónimos y nuevos registros, así como una lista de los sabélidos nombrados por el Prof. Ernst Ehlers. Se enlistan 13 géneros y 23 especies para Chile. Fabriciinae está representada por una especie y Sabellinae por 12 géneros y 22 especies. Tres especies que fueron descritas originalmente para Chile actualmente no se consideran válidas, mientras que siete registros permanecen como cuestionables hasta que una revisión garantice su distribución. El Prof. Ehlers estableció el género Potamis y 24 especies de sabélidos para diversas localidades del mundo. Entre esas especies, 17 se consideran válidas, dos insertae sedis y cinco han sido sinonimizadas, mientras que el género Potamis fue reemplazado con Potamethus. Se incluye una clave ilustrada para los géneros de Sabellinae.
\end{abstract}

Palabras clave: Sabellidae, Chile, Ehlers, listado, gusanos plumero, clave.

1 Geomare, A. C., Av. Miguel Alemán 616-4B, Col. Lázaro Cárdenas, 82040, Mazatlán, Sinaloa, México. maria_ana_tovar@ yahoo.com 


\section{ABSTRACT}

The most recent checklist of polychaetes of Chile dates from 1985. Since then systematic contributions for the family Sabellidae have been published resulting in changes in the taxonomic status of various genera and species. This contribution provides new names, synonymies and new records. A list of sabellid taxa named by Prof. Ernst Ehlers is also included. Thirteen genera and 23 species are listed for Chile. Fabriciinae is represented by one species and Sabellinae with 12 genera and 22 species. Three species that were originally described from Chile are currently recognised as not valid, and seven records as questionable until any revision sustains their distribution. Prof. Ehlers established 24 species of sabellid worms from several worldwide localities and the genus Potamis. Among these species, 17 are currently accepted names, two are insertae sedis and five have been synonymized, while the genus name Potamis was replaced with Potamethus. An illustrated key is included for the genera of Sabellinae.

Key words: Sabellidae, Chile, Ehlers, checklist, fan worms, key.

\section{INTRODUCTION}

Sabellidae Latreille, 1825 is a family of polychaetous annelids commonly known as fan worms, feather-duster worms, or sea flowers. Living specimens are easily recognized due to their often colourful crown frequently projecting from the mouth of their tubes. Rioja (1923) divided the sabellids into three subfamilies based primarily on chaetal characters: Sabellinae Latreille, 1825, Myxicolinae Renier in Meneghini, 1847, and Fabriciinae Rioja, 1923. Fitzhugh's (1989) morphology-based cladistic analysis resulted in significant changes to the previous classification of Sabellidae as it demonstrated that only the subfamilies Fabriciinae and Sabellinae could be accepted as monophyletic.

Kupriyanova \& Rouse (2008) studied the monophyly of the Sabellidae based on molecular data from three nuclear genes of sabellins and fabriciins. Their molecular evidence supports the hypothesis that the Sabellidae does not constitute a monophyletic group as currently formulated since it contains the Serpulidae. Additionally, the Fabriciinae were found to be more closely related to the Serpulidae than to Sabellinae. The authors therefore emphasized that the Fabriciinae must be removed from the Sabellidae and be referred to the Fabriciidae, with the revised Sabellidae being equal to the previous subfamily Sabellinae because its represents the simplest solution with the least disruption to current nomenclature. Recently, Capa et al. (2010) based on morphology and multiple genes (nuclear ribosomal RNA genes $18 \mathrm{~S}$ and $28 \mathrm{~S}$ and mitochondrial gene
16S) also confirmed the monophyly of Fabriciinae and Serpulidae, being Serpulidae the sister group to Fabriciinae. This study follows the traditional recognition of the subfamilies Fabriciinae and Sabellinae until the new classification, with the recognition of Fabriciidae as family, will be re-organized according the rules of the International Code of Zoological Nomenclature (1999).

In Chile, a number of important studies on polychaetes have been carried out. These works are summarized in the checklist of Rozbaczylo (1985), in which, 29 species of sabellids belonging to 16 genera are listed. On the other hand, Prof. Ernst Ehlers did a great contribution to the study of sabellid polychaetes, as shows his 24 detailed descriptions of feather duster worms and beautiful illustrations. The objectives of this contribution are to provide updated information of the Sabellidae species currently known to occur in Chile; to include the sabellid taxa named by Prof. Ehlers as a modest homage to his great contribution to the study of Sabellidae, and to provide a key for Sabellinae.

\section{MATERIAL AND METHODS}

The sabellid genera described or recorded in Chile are given in alphabetic order, followed by an alphabetic list of species. The nomenclatural information includes the original name, year of publication, pages, plates and figures. Data for the type locality as complete as available in the original description are also included. Data for type materials are provided as complete as available in museums 
data bases or original descriptions. A question mark symbol (?) indicates incomplete or unavailable information. The following abbreviations correspond to repository institutions:

AM: Australian Museum, Sydney, Australia. Sweden

GNM: Göteborgs Naturhistoriska Museum,

LACM-AHF: Los Angeles County Museum of Natural History, Allan Hancock Foundation, Los Angeles, California, USA.

NHMW: Naturhistorisches Museum Wien, Wien, Austria.

NRS: Naturhistorika Rikmusset Stockholm, Sweden.

RSM: Royal Scottish Museum Edinburgh, Scotland.

USNM: The Natural History Museum, Smithsonian Institution, Washington DC, USA.

$\mathrm{ZMH}$ : Zoologisches Institut und Museum, Univ. Hamburg, Hamburg, Germany.

ZMHUB: Zoological Museum, Humboldt University, Berlin, Germany.

ZMO: Zoological Museum, University of Oslo, Norway.

Although synonyms are provided, a list of the species not recognized as valid is given with their nomenclatural reference, type locality and remarks to facilitate further searches. Some questionable records in Chile are included until revision upholds their distribution.

Sabellid worms erected by Ernst Ehlers were included (currently accepted names, unaccepted names or insertae sedis names are specified). An illustrate key to genera in Sabellinae is presented here (a key for Fabriciinae was provided by Fitzhugh, 1998). At the end of this paper, the complete literature references of the consulted publications are provided.

\section{RESULTS}

Thirteen genera and 23 species are listed for Chile. Fabriciinae is represented by one species and Sabellinae with 22 species and 12 genera. Three species that were originally described from Chile are currently recognised as not valid, and seven records as questionable until any revision sustains their distribution.
SABELLID TAXA RECORDED OR DESCRIBED

FOR CHILE: currently accepted names

GENUS Amphicorina Claparède, 1864

Amphicorina Claparède, 1864: 474.--Rouse, 1994: 202._- Giangrande et al. 1999: 195-196.Nogueira \& Amaral, 2000: 618.

Oria de Quatrefages, 1866: 462.- Banse, 1957: 68

Oriopsis Caullery \& Mesnil, 1896: 483-484, Figs 1-2.-Banse, 1957: 68-70.- Fitzhugh, 1989: 66-67.- Rouse, 1994: 181.

Oridia Rioja, 1917: 73.-- Banse, 1957: 68.

Oriades Chamberlin, 1919: 470.- Banse, 1957: 68

1) Amphicorina alata (Ehlers, 1897)

Fabricia alata Ehlers, 1897: 135-137, Pl. 9, Figs 206-210.- Ehlers, 1901b: 218.- Hartman, 1953: 11, Fig. 21a-e.- Rullier, 1954: 25, 27.

Oria alata.-Zenkevitch, 1925: 44.

Oriopsis alata.- Banse, 1957: 73-74, Fig. 2a-c.- Hartman, 1966: 119, 121, Pl. 40, Figs. 8-10.- Knight-Jones \& Bowden, 1984: 813.

Amphicorina alata.- Giangrande et al. 1999: 197.

Type locality: Ushuaia, Beagle Channel, 1.82-3.65 m depth.

Type material: ZMH V-4955-57 (holotype).

Records: See Rozbaczylo (1985: 209) as Oriopsis.

\section{2) Amphicorina alatoides}

(Hartmann-Schröder, 1962)

Oriopsis alatoides Hartmann-Schröder, 1962: 165, Figs 222-224.

Amphicorina alatoides.- Giangrande et al. 1999: 197.

Type locality: Chile, Huasco.

Type material: ZMH P-15241(holotype).

Records: Only known for the type locality.

3) Amphicorina limbata (Ehlers, 1897)

Oria limbata Ehlers, 1897: 137-139, Pl. 9, Figs. 211-216. - Ehlers, 1901b: 218.- Fauvel, 1916: 476-477. 
Oridia limbata.- Augener, 1926: 269.Benham, 1927: 130.- Hartman, 1951: 381.Hartman, 1953: 54-55, Fig. 20a-d.

Oriopsis limbata.- Banse, 1957: 76-78, Fig. 3a-e.- Hartman, 1966: 121, Pl. 41, Figs. 3-6.- Hartman, 1967: 174.

Amphicorina limbata.- Giangrande et al. 1999: 197. $\mathrm{m}$ depth.

Type locality: Ushuaia, Beagle Channel, 9.14

Type material: ZMH V-4958.

Records: See Rozbaczylo (1985: 210) as Oriopsis.

4) Amphicorina magellanica

(Hartmann-Schröder, 1962)

Oriopsis magellanica Hartmann-Schröder, 1962: 163, Figs. 216-218.

Type locality: Punta Arenas, Chile, in Macrocystis holdfasts, 3-4 m depth.

Type material: ZMH P-15230 (holotype and 4 paratypes).

Records for Chile: See Rozbaczylo (1985: 210) as Oriopsis.

5) Amphicorina taltalensis

(Hartmann-Schröder, 1962)

Oriopsis taltalensis Hartmann-Schröder, 1962: 163-164, Figs 219-221.

Amphicorina taltanensis.- Giangrande et al. 1999: 197.

Type locality: Chile, $4 \mathrm{~km} \mathrm{~N}$ Taltal.

Type material: ZMH P-15240 (holotype).

Records: Only known for the type locality.

GENUS Branchiomma Kölliker, 1859

Branchiomma Kölliker, 1859: 537.- TovarHernández \& Knight-Jones, 2006: 5.

Dasychone Sars, 1862: 118.- Hartman, 1959: 537.

Dasychonopsis Bush, 1905: 198.-Hartman, 1959: 537.

6) Branchiomma curtum (Ehlers, 1901a)

Dasychone curta Ehlers, 1901a: 216-218, Pl. 25, Figs. 10-13.-Ehlers, 1901b: 268.-Ehlers, 1907: 28.
Dasychone cingulata (Grube) var. curta Ehlers.- Augener, 1922a: 211.-Benham, 1927: 137-139, Pl. 4, Figs 124-125.

Branchiomma curtum.- Tovar-Hernández \& Knight-Jones, 2006: 20-21, 23-24, Figs 5A-G, 8D-E, 9H-J, 10F, 11E.

Type locality: Chile, Masatierra, Juan Fernandez Islands, $33.6 \mathrm{~m}$ depth.

Type material: ZMHUB 376 (syntypes). locality.

Records: In Chile, only known for the type

Remarks: At least four species of Branchiomma have been considered as alien (Tovar-Hernández et al. 2009), these include B. luctuosum (Grube), B. boholense (Grube), B. curtum and B. bairdi (McIntosh). Among these species, the linear distribution of Branchiomma curtum from Chile, New Zealand, Cape Verde Islands and Mexico may have been assisted by ballast water from ships (Tovar-Hernández \& Knight-Jones 2006).

\section{GENUS Chone Krøyer, 1856}

Chone Krøyer, 1856: 13.-_ Sars, 1862: 119.Malmgren, 1866: 404.—McIntosh, 1923: 287.-Day, 1967: 776.-Banse, 1972: 460.-Fitzhugh, 1989: 67.- Giangrande, 1992: 518. - Tovar-Hernández \& Sosa-Rodríguez, 2006: 36-37.- Tovar-Hernández, 2008: 2216-2218.

Parachonia Kinberg, 1867: 355.-Johansson, 1925: 27-28.

Megachone Johnson, 1901: 430.- Banse, 1972: 460.

Metachone Bush in Moore, 1904: 190.Fauvel, 1927: 334.

7) Chone rosea Hartmann-Schröder, 1965

Chone rosea Hartmann-Schröder, 1965: 276-278, Figs 279-280.- - Tovar-Hernández, 2007. 55-56, Fig. 14.

Type locality: off south Chile, Punta Galera, Valdivia, $260 \mathrm{~m}$ depth.

Type material: ZMH P-15202 (holotype).

Records: See Rozbaczylo (1985: 207).

8) Chone striata Hartmann-Schröder, 1965

Chone striata Hartmann-Schröder, 1965: 278-280, Figs 281-283. 
Type locality: Chile, Puerto Inglés, Bahía de Ancud, $12 \mathrm{~m}$ depth.

Type material: ZMH P-15203 (holotype and one paratype).

Records: Only known for the type locality.

GENUS Euchone Malmgren, 1866

Euchone Malmgren, 1866: 405-406.-Banse, 1972: 481-482.— Fitzhugh, 1989: 68.

9) Euchone pallida Ehlers, 1908

Euchone pallida Ehlers, 1908: 159, Pl. 21, Figs. 10-15, Pl. 22, Figs. 1-4.- Monro, 1930: 203-204.- Hartman, 1966: 117, Pl. 49, Figs. 11-15.-- Hartman, 1967: 172.- Hartman, 1978: 203, 205-206.- Hartmann-Schröder \& Rosenfeldt, 1991: 89.-- Licciano et al. 2009: 30-32, Fig. 1.

Type locality: East of Kerguelen Islands, Antarctica.

Type material:?

Records for Chile: See Rozbaczylo (1985: 208).

GENUS Jasmineira Langerhans, 1880

Jasmineira Langerhans, 1880: 113.— Day, 1967: 779.-_Fitzhugh, 1989: 68-69.- HartmannSchröder, 1996: 556.- Fitzhugh, 2002: 383.

10) Jasmineira regularis Hartman, 1978

Jasmineria regularis Hartman, 1978: 206-

208, Figs 41a-g.

Type locality: Weddell Sea, 650 m depth.

Type material: USNM 47095 (holotype).

Records: See Rozbaczylo (1985: 209).

GENUS Notaulax Tauber, 1879

Hypsicomus Grube, 1870: 348 (in part; not Sabella stichophthalmos Grube).- Fauvel, 1927: 312.- Hartmann-Schröder, 1971: 502.

Notaulax Tauber, 1879: 136.- Levinsen, 1883: 185, 187-188.—Perkins, 1984: 327, 329.Fitzhugh, 1989: 75.

Protulides Webster, 1884: 325.- Perkins, 1984: 327.

Eurato de Saint-Joseph, 1894: 219-220.Perkins, 1984: 327.
Hypsicomatopsis Augener, 1922b: 49.Perkins, 1984: 327.

11) Notaulax tilosaula (Schmarda, 1861)

Sabella tilosaula Schmarda, 1861: 34, Pl. 23, Fig. 191.- Ehlers, 1901b: 216.

Notaulax tilosaula.-Perkins, 1984: 328.

Type locality: Coast of Chile.

Type material: ?

Records: Only known for the type locality.

Remarks: Hartman (1959: 565) stated that this species is possibly Sabella phaeotenia Schmarda (both currently recognized in Notaulax), a species described from Ceylon, however Perkins (1984) recognized both as different species. Although the species is only known for the type locality, the record of Notaulax phaeotenia (Schmarda) from the South Patagonian Icefield by Montiel et al. (2004) could be assigned to $N$. tilosaula. See remarks of Notaulax phaeotenia in page 14.

GENUS Novafabricia Fitzhugh, 1990

12) Novafabricia chilensis

(Hartmann-Schröder, 1962)

Fabriciola chilensis Hartmann-Schröder, 1962: 161, Figs. 212-215.- Knight-Jones \& Bowden, 1984: 814.

Novafabricia chilensis. - Fitzhugh, 1990c: 9-10, Fig. 6.

Type locality: Chile, Arica, in rhizoids of Macrocystis.

Type material: ZMH P-15224 (holotype), P-15225 (10 paratypes).

Records: See Rozbaczylo (1985: 208).

GENUS Parasabella Bush, 1905

= Demonax Kinberg, 1867: 354 (not Thomson, 1860); 1910: 72.- Johansson, 1925: 26-27; 1927: 136.-Knight-Jones, 1983: 254.-Perkins, 1984: 292-293.- Knight-Jones \& Walker, 1985: 605.- Fitzhugh, 1989: 75-76.- Giangrande, 1994: 229-230.

Parasabella Bush, 1905: 191, 199-200.Johansson, 1927: 136.- Tovar-Hernández \& Harris, 2010: 14.

Distylidia Hartman, 1961: 129._- Fauchald, 1977: 138._- Banse, 1979: 870. 
Remarks: Kinberg (1867) described the new genus Demonax for four new species of sabellid polychaetes. This name is a junior homonym of Demonax Thomson, 1860, a genus of round necked longhorn beetles (Insecta: Coleoptera: Cerambycidae). Tovar-Hernández \& Harris (2010) reintroduced the genus Parasabella Bush, 1905 for the replacement of Demonax Kinberg, 1867, since a junior homonym must be rejected and replaced either by an available and potentially valid synonym (Art. 23.3.5) or, for lack of such name, by a new substitute name (Article 60 of the International Code of Zoological Nomenclature, 1999).

13) Parasabella fernandezensis (Augener, 1922) Sabella fernandezensis Augener, 1922a: 210-211, Textfigs. 10a-b.

Demonax fernandezensis.- Knight-Jones \& Perkins, 1998: 404.

Parasabella fernandezensis.- Tovar-Hernández \& Harris, 2010: 15.

Type locality: Chile, Juan Fernandez Island (Robinson Crusoe), 30-40 m depth.

Type material: GNM 1534.

Records: Only known for the type locality.

14) Parasabella leucaspis (Kinberg, 1867)

Demonax leucaspis Kinberg, 1867: 354.Johansson, 1925: 24, Fig. 8, no. 7-11; 1927: 126, Text-fig. 14, no. 4, 6-7.-- Knight-Jones, 1983: 256-257.- Perkins, 1984: 296-299, Figs. 3-5.

Demonax incertus Kinberg, 1867: 354.- Johansson, 1925: 22, 24, Fig. 8, no. 1-6.-- Hartman, 1959: 541.-- Perkins, 1984: 296.

Demonax leucaspius.- Johansson, 1925: 24, Fig. 8, no. 7-11.

Parasabella leucaspis.- Tovar-Hernández \& Harris, 2010: 15.

Type locality: Perú, Callao, Isla San Lorenzo, intertidal.

Type material: NRS (Fauchald, 2007)

Records: See Rozbaczylo (1985: 207).

Remarks: Parasabella incertus was described by Kinberg (1867: 354) from Valparaíso to 3.6-5.5 m depth (as Demonax). Johansson (1925, 1927) suggested $P$. incertus as synonym of $P$. leucaspis as corroborated later by Perkins, 1984: 296.
GENUS Perkinsiana Knight-Jones, 1983

Perkinsiana Knight-Jones, 1983: 273-274.Rouse, 1996: 102-103.- Giangrande \& Gambi, 1997: 268.- Capa, 2007: 549.

15) Perkinsiana antarctica (Kinberg, 1867)

Laonome antarctica Kinberg, 1867: 354.Ehlers, 1901a: 17.

Potamilla antarctica.- Monro, 1930: 199-201.- Augener, 1932: 68.- Fauvel, 1936: 37.- Monro, 1936: 188.- - Hartman, 1953: 53.Hartman, 1966: 125-126, Pl. 41, Figs. 10-12.

Perkinsiana antarctica.- Knight-Jones, 1983: 277-279, Fig. 14.— Hartmann-Schröder \& Rosenfeldt, 1991: 88.- Giangrande \& Gambi, 1997: 268, Figs. 1-2.- Rios et al. 2007: 94.

Type locality: Bahía Laredo, Punta Arenas, Strait of Magellan, Chile.

Type material: AM W 233505 (neotype).

Records: See Rozbaczylo (1985: 210-211). Bahia Laredo, Magellan Strait, associated to the holdfasts of the kelp Macrocystis pyrifera (Rios et al. 2007: 94).

Remarks: Perkinsiana antarctica was considered the most common sabellid in Antarctic and subantarctic waters. It was poorly described and no illustrations were provided but their records from Antarctic and subantarctic areas are extensive (see Giangrande \& Gambi (1997) and references therein) showing a high degree of morphological variability. The revision by Giangrande \& Gambi (1997) includes the designation of a neotype from Punta Arenas (Chile) and their study revealed that the species has been often confused with other taxa and that probably $P$. antarctica has a distribution restricted to subantarctic areas.

16) Perkinsiana corcovandensis

(Hartmann-Schröder, 1965)

Potamilla corcovandensis Hartmann-Schröder, 1965: 269-271, Figs. 271-272.

Perkinsiana corcovadensis.- Knight-Jones, 1983: 287-288.

Type locality: Corcorvado Gulf, South Chile, 190 m depth.

Type material: ZMH P-15224 (holotype).

Records: Only known for the type locality. 
17) Perkinsiana littoralis (Hartman, 1967)

Potamethus littoralis Hartman, 1967: 174175, Pl. 51.

Perkinsiana littoralis. - Knight-Jones, 1983: 288-289, fig. 20.- Giangrande \& Gambi, 1997: 268, Figs. 3-4. depth.

Type locality: South Shetlands Island, 79 m

Type material: USNM 55570 (holotype).

Records: See Rozbaczylo (1985).

18) Perkinsiana magalhaensis (Kinberg, 1867)

Sabella magalhaensis Kinberg, 1867: 353.Ehlers, 1901b: 215.- Kinberg, 1910: 72, Pl. 27, Fig. 7.- Johansson, 1925: 22, Fig. 7.

Bispira magalhaensis.- Fauvel, 1916: 471, Pl. 9, Figs. 34-43.- Monro, 1930: 201.-Hartman, 1966: 123, Pl. 39, Figs 2-6.

Type locality: Isla Sánchez (Bucket Island),

Bahía San Nicolás, Strait of Magellan.

Type material: NRS 1086 (holotype?).

Records: in Chile only known for the type locality.

19) Perkinsiana pusilla (Johansson, 1922)

Sabella pusilla Johansson, 1922: 5-6, Pl. 1, Fig. 6.- Hartman, 1966: 127, Pl. 42, Fig. 9.

Perkinsiana pusilla.- Knight-Jones \& Perkins, 1998: 404.

Type locality: Falkland Islands, Puerto Williams, $12 \mathrm{~m}$ depth and Tierra del Fuego, E Ushuaia, 10 $m$ depth.

Type material: NRS 1084 (holotype?).

Records: See Rozbaczylo (1985: 213).

GENUS Potamethus Chamberlin, 1919

Potamis Ehlers, 1887: 278.

Potamethus Chamberlin, 1919: 469._-KnightJones, 1983: 269._- Fitzhugh, 1989: 71.

20) Potamethus scotiae (Pixell, 1913)

Potamis scotiae Pixell, 1913: 356-357, Fig. 7.

Potamethus scotiae.-Hartman, 1966: 123, Pl. 42, Figs. 1-4.--Hartman, 1967: 175.-1978: 208, Figs. 42a-e.-Knight-Jones, 1983: 271, Fig. 11L-N.

Type locality: off Princess Martha Coast, Antarctic Ocean ( $\left.71^{\circ} 22^{\prime} \mathrm{S}, 16^{\circ} 34^{\prime} \mathrm{W}\right), 2578.6$ $\mathrm{m}$ depth.
Type material: RSM 1921.143.1486 (holotype).

Records: See Rozbaczylo (1985: 211).

GENUS Potamilla Malmgren, 1866

21) Potamilla antarctica

christenseni Augener, 1932

Potamilla antarctica christenseni Augener, 1932: 69-70.- Hartman, 1966: 126.

Type locality: Chile, Adelaide Island, 620 $\mathrm{m}$ depth

Type material: ZMO C547 (holotype).

Records: Only known for the type locality.

Remarks: Augener (1932) described Potamilla antarctica christenseni from the Adelaide Island but did not provide illustrations. Giangrande \& Gambi (1997) examined the holotype (ZMO, C547) and revealed that it belongs to a different genus, probably Notaulax.

GENUS Pseudopotamilla Bush, 1905

Pseudopotamilla Bush, 1905: 203.-KnightJones, 1983: 253-254.- Capa, 2007: 555-556.

22) Pseudopotamilla polyophthalma Hartmann-Schröder, 1965

Pseudopotamilla polyophthalma HartmannSchröder, 1965: 271-273, Figs. 273-275.

Type locality: Chile, Punta Lavapié, $38^{\circ} 08.7^{\prime}$ S, $73^{\circ} 38.6^{\prime} \mathrm{W}, 58 \mathrm{~m}$ depth.

Type material: ZMH P-15243 (holotype).

Records: In Chile only known for the type locality.

GENUS Terebrasabella Fitzhugh \& Rouse, 1999

Terebrasabella Fitzhugh \& Rouse, 1999: 358-359.- Murray \& Rouse, 2007: 52-53.

23) Terebrasabella heterouncinata Fitzhugh \& Rouse, 1999

Terebrasabella heterouncinata Fitzhugh \& Rouse, 1999: 359-367, Figs. 1-11.

Type locality: Sudáfrica, Playa Seaforth, Simonstown (34 $\left.12^{\prime} \mathrm{S}, 18^{\circ} 27^{\prime} \mathrm{E}\right)$.

Type material: LACM-AHF 1908 (holotype), LACM-AHF 1909 (paratypes).

Records: Puerto Montt, in farms of the red abalone Haliotis rufescens (Moreno et al. 2006). 
SABELLID TAXA DESCRIBED FOR

CHILE: unaccepted names

1) Demonax incertus Kinberg, 1867: 354.

Type locality: Valparaíso, 3.65-5.48 m depth.

Remarks: Parasabella was reintroduced as a

replacement name for Demonax (Tovar-Hernández \& Harris 2010). Johansson $(1925,1927)$ suggested that $P$. incertus (as Demonax) is a synonym of $P$. leucaspis as corroborated later by Perkins, 1984: 296.

2) Demonax tilosaulus Kinberg, 1867: 354.

Type locality: Chile.

Remarks: Kinberg (1867) originally described four species in Demonax (now Parasabella). One species incorrectly reported by him as Demonax tilosaulus (not Sabella tilosaula Schmarda, 1861) is a Chone species according to Hartman (1959: 514).

3) Megalomma monoculata HartmanSchröder, 1965: 273-276, Figs. 276-278.

Type locality: Chile, Punta Topocalma $\left(34^{\circ}\right.$ 08.2' S, $\left.72^{\circ} 09.1^{\prime} \mathrm{W}\right), 160 \mathrm{~m}$ depth, $11.3^{\circ} \mathrm{C}, 0.23$ $\mathrm{ml} / 1 \mathrm{O}_{2}$.

Remarks: Knight-Jones (1997) synonymized M. monoculata with M. pigmentum Reish. Both species have caruncle, peristomium exposed dorsally and laterally above collar, eyes only in the dorsalmost radioles and similar chaetae and uncini.

\section{SABELLID TAXA WITH QUESTIONABLE DISTRIBUTION IN CHILE}

1) Amphicorina ehlersi (Day, 1961)

Oriopsis ehlersi Day, 1961: 546-547, Fig. 16a-g.- Knight-Jones \& Bowden, 1984: 813.

Type locality: South Africa, False Bay, 14-17 $\mathrm{m}$ depth.

Remarks: Knight-Jones \& Bowden (1984) recorded Amphicorina ehlersi (as Oriopsis) from the Chilean shores between Iñaque and Concepción. These authors mentioned that the webbing is less conspicuous in materials from Chile than those recorded in Day's description but examination of chaetae and uncini are needed.
2) Amphiglena pacifica Annenkova, 1934

Amphiglene pacifica Annenkova, 1934:

328-329, Fig. 9a-e.

Amphiglena pacifica.- Kohn \& Lloyd, 1973: 604.

Type locality: Bering Strait.

3) Euchone analis (Krøyer, 1856)

Sabella analis Krøyer, 1856: 17.

84-85.

Euchone analis.- Augener, 1932: 70-71,

Type locality: Greenland.

4) Fabricia sabella (Ehrenberg, 1836)

Amphicora sabella Ehrenberg, 1836: 4.

Fabricia sabella.- Kohn \& Lloyd, 1973:

704.- Hartman, 1967: 173.

Type locality: Western Europe.

5) Notaulax phaeotenia (Schmarda, 1861)

Sabella phaeotenia Schmarda, 1861: 35, pl. 22, fig. 188.

Hyspicomus phaeotenia.- Hartmann-Schröder, 1983: 272.- Montiel et al. 2004: 61, Figs. 8-9.

Notaulax phaeotenia.-Perkins, 1984: 328.

Remarks: Hartman (1959) suggested that Hypsicomus tilosaula Schmarda is possibly Sabella phaeotenia Schmarda (both currently recognized in Notaulax), a species from Ceylon; however Perkins (1984) recognized both as different species. Although examination of type materials of both species is certainly needed, the comparison of original drawings by Schmarda for N. phaeotaenia (plate 22 fig. 188) and $N$. tilosaula (plate 23, fig. 191) allow differentiate both species: the branchial crown of $N$. phaeotaenia have red and yellow alternated bands and a basal lamina of the branchial crown long while in $N$. tilosaula the crown is homogeneously red and the basal lamina is short. Montiel et al. (2004) recorded Hypsicomus phaeotenia from the South Patagonian Icefield at $75 \mathrm{~m}$ depth and provided illustrations of anterior thorax and base of the branchial crown, the basal lamina is short (as N. tilosaula).

6) Potamilla neglecta (Sars, 1851)

Sabella neglecta Sars, 1851: 203.

Potamilla neglecta.- Hartman, 1953: 11.

Type locality: Trømso, Norway. 
7) Sabella pavonina Savigny, 1822

Sabella pavonina Savigny, 1822: 79-80.Augener, 1932: 67-68.

Type locality: Southern Europe.

\section{SABELLID TAXA ERECTED BY PROF. ERNST EHLERS}

Prof. Ehlers established 24 species of sabellid worms from several worldwide localities and the genus Potamis. Among these species, 17 are currently accepted names (*), five have been synonymized (**) and two are insertae sedis (***), while the genus name Potamis was replaced with Potamethus. Furthermore, two species of sabellids were named in honour of Ehlers: Oriopsis ehlersi Day, 1961 and Potamilla ehlersi Gravier, 1906. Oriopsis ehlersi was described from South Africa and currently is recognized as valid within the genus Amphicorina. Potamilla ehlersi was described from the Red Sea and recognized as synonym of Pseudopotamilla saxicava (de Quatrefages, 1866) as suggested by Knight-Jones et al. in a poster presented during the $8^{\text {th }}$ International Polychaete Conference in Madrid. Dr. Knight-Jones was working on the reestablishment of $P$. saxicava; unfortunately she died before she could conclude a full revision of the genus Pseudopotamilla.

1) *Amphicorina alata (Ehlers, 1897) (see page 9)

2) *Amphicorina limbata (Ehlers, 1897) (see pages 9-10)

3) *Amphicorina parvula (Ehlers, 1913)

Oria parvula Ehlers, 1913: 580-581.

Amphicorina parvula.- Giangrande et al. 1999: 197.

Type locality: Simonstown, South Africa.

Type material: ZMB lost (Fauchald, 2007).

4) **Banchiomma conspersum (Ehlers, 1887)

Dasychone conspersa Ehlers, 1887: 266-270,

Pl. 54, Figs. 1-6.

Dasychonopsis arenosa Treadwell, 1924: 1-2, Figs. 1-4.- Tovar-Hernández \& Knight-Jones, 2006: 18.
Branchiomma conspersum.-Knight-Jones, 1994: 192.- Tovar-Hernández \& Knight-Jones, 2006: 18-20, Figs. 4A-J, 10E, 11D.

Type locality: Florida, Key West, 1.68-3.36 m depth.

Type material: Ehler's (1887) syntypes of Dasychone conspersa were deposited in the Museum of Comparative Zoology and the Zoological Museum of the Humboldt University Berlin. The single specimen in MCZ 848, which had been subsequently wrongly labelled as a holotype, had dried out by 1937 and is now missing (Ardis Johnston pers. com). A lectotype was selected from Ehlers's other syntypes, ZMHUB 6791 by Phyllis Knight-Jones. There were 5 units in the vial, two of these (one with crown attached to body and one crown without body) agree with Ehler's description in having stylodes as wide as or wider than the rachis. The more complete specimen was therefore chosen as lectotype (Tovar-Hernández \& Knight-Jones 2006). Unfortunately, Ehlers's figure (pl. 54, fig. 3) of a radiole with stylodes is in side view, and the width of the macrostylodes does not show. That figure of the radiole may have misled subsequent sabellid workers to synonymise $B$. conspersum with $B$. bairdi. One of the syntypes was indeed Branchiomma bairdi (now ZMHUB 11064) but the proportions of Ehlers' figured thorax and collar, agree best with the lectotype. Two other syntypes were yet other species, Branchiomma nigromaculatum and Bispira melanostigma (now ZMHUB 11063 and 11062 respectively).

Remarks: Gravier (1908) recorded B. conspersum (as D. conspersa) from Djibouti, Red Sea, but his material (MNHN A257) is B. boholense (Grube) a species in which thorax and stylode proportions resemble $B$. bairdi rather than $B$. conspersum (Tovar-Hernández \& Knight-Jones 2006). As Gravier's (1908) Red Sea record is another species of Branchiomma, B. conspersum has not been found outside the Grand Caribbean.

5) **Banchiomma corolliferum

(Ehlers, 1913) re-established

Dasychone corollifera Ehlers, 1913: 571-572,

Pl. 45, Figs. 1-7.

Branchiomma corolliferum.- Knight-Jones, 1994: 192

Type locality: South Africa, Simonstown. Type material: ZMHUB 5921 (holotype). 
Remarks: Hartman (1959: 540) suggested that $D$. corollifera is a subjective synonym of $B$. nigromaculatum but no comparison of both type materials were done. Day's (1967) figure of Branchiomma nigromaculatum from South Africa, with very enlarged basal stylodes, is unlike those of $B$. nigromaculatum and according to material from Saint-James, East coast of South Africa it is likely to be B. coralliferum (Ehlers, 1913), a well figured species which Day (1955) wrongly synonymized with $B$. nigromaculatum (TovarHernández \& Knight-Jones 2006).

6) **Banchiomma curtum (Ehlers, 1901) (see pages 10).

7) **Banchiomma hypsilophum Ehlers,

1920: 67, Pl. 3, Figs. 13-15.

Type locality: Amboina, Moluccas Islands, Indonesia.

Type material: ZMHP-E 1302 (original material).

Remarks: A junior synonym of Stylomma palmata de Quatrefages fide Knight-Jones, 1997: 322.

8) **Dasychone foliosa Ehlers, 1913:

572-574, Pl. 45, Figs. 8-16.

Type locality: Simonstown, South Africa.

Type material: ?

Remarks: A junior synonym of Notaulax phaeotaenia (Schmarda). Dasychone foliosa Ehlers, was synonymyzed by Hartman (1959: 540) with Sabella violacea Schmarda, a species from the Cape of Good Hope, South Africa. Later, Hartman (1965: 78) synonymized S. violacea with Hypsicomus phaeotaenia Schmarda, a species described from Ceylon. Hypsicomus phaetoenia is currently recognized in the genus Notaulax (Perkins 1984: 328).

9) **Euchone pallida Ehlers, 1908 (see page 11).

10) ***Euchone rubella Ehlers, 1871

Euchone rubella Ehlers, 1871: 82.

Type locality: Spitsbergen.

Type material: ?

Remarks: Hartman (1959: 543) listed Euchone rubella as being a subjective synonym of Euchone analis Krøyer, apparently only based on that both species were described from the same type locality (Spitsbergen). Location of type material of Euchone rubella is unknown.
11) **Eudistylia brevicomata (Ehlers, 1905) Sabella (Potamilla) brevicomara Ehlers, 1905: 298.

Eudistylia brevicomata.- Hartman, 1959:

543.- Banse, 1979: 843.

Type locality: Bare Island, New Zealand.

Type material: ZMH P-E 1334 (holotype).

12) **Jasmineira analis Ehlers, 1908

Jasmineira analis Ehlers, 1908: 48.

Type locality: Atlantic Ocean, Southwest Africa, off Namibia.

Type material: ZMH P-1329 (holotype).

Remarks: This species probably does not belong in this genus (Day 1967: 779; Fitzhugh 2002: 387).

13) ***Jasmineira caeca Ehlers, 1913

Jasmineira caeca Ehlers, 1913: 579.- Hart-

man, 1966: 119, Pl. 40, Figs. 1-3.

Jasmineira scotti Benham, 1927: 131, Pl 3,

Figs 100-107.-- Hartman, 1966: 119.

Type locality: Kerguelen Islands, Observatory Bai.

Type material: ?

14) **Jasmineira macrophthalma Ehlers, 1913

Jasmineira macrophthalma Ehlers, 1913:

578-579.- - Hartman, 1966: 119.

Type locality: Kerguelen Islands, Observatory Bai.

Type material: ?

15) ***Laonome ceratodaula Ehlers, 1905

Laonome ceratodaula Ehlers, 1905: 64.

Type locality: New Zealand.

Type material: ?

Remarks: Hartman (1959: 548) listed Laonome ceratodaula Ehlers as being a subjective synonym of Sabellastarte indica Savigny, although no comparisons or comments were provided. In the revision of the genus Sabellastarte, Knight-Jones \& Mackie (2003) stated that Sabellastarte indica Savigny is junior to Sabella indica Abildgaard in Pectinariidae, consequently, the accepted name for S. indica is Sabellastarte spectabilis (Grube). However, Knight-Jones \& Mackie (2003) did not examined type material of $L$. ceratodaula, these are not in the $\mathrm{ZHH}$ neither in $\mathrm{ZMHUB}$. 
16) **:Megalomma bioculatum (Ehlers, 1887)

Branchiomma bioculatum Ehlers, 1887: 260-263, Pl. 53, Figs. 1-9.

Megalomma bioculatum.- Johansson, 1927:

132.- Perkins, 1984: 352-354, Fig. 38.

Type locality: Straits off Florida, $24^{\circ} 37.5^{\prime} \mathrm{N}$, $83^{\circ} 36^{\prime} \mathrm{W}, 166 \mathrm{~m}$ depth.

Type material: MCZ 669 (holotype and six syntypes partially dried, incomplete, only crowns with anterior thorax). MCZ 824 (lot with an onuphid and an oenonid from the original series of syntypes).

17) **Megalomma lobiferum (Ehlers, 1887)

Branchiomma lobiferum Ehlers, 1887: 254259, Pl. 53, Figs. 10-15.

Megalomma lobiferum.- Johansson, 1927: 132.- - Hartman, 1959: 550.- Perkins, 1984: 354-357, Figs. 39-40. - Tovar-Hernández \& SalazarVallejo, 2006: 42-43, Fig. 10.- Tovar-Hernández \& Salazar-Vallejo, 2008: 1961, 1963-1964, Figs 3-5. m depth.

Type locality: Florida, Key West, 1.82-3.6

Type material: MCZ 835 (holotype).

18) *"Megalomma suspiciens (Ehlers, 1904)

Branchiomma suspiciens Ehlers, 1904: 62-63, Pl. 9, Figs. 1-6.

Megalomma suspiciens.- Hartman, 1959:

550.—Knight-Jones, 1997: 314._- Glasby \& Read, 1998: 359.

Type locality: New Zealand, French Pass.

Type material: O-ZMUH PE 1034-6 (holotype).

19) **Notaulax circumspiciens (Ehlers, 1887)

Hypsicomus circumspiciens Ehlers, 1887:

271-277, Pl. 55, Figs 5-13, Pl. 56, Figs. 1-3.

Notaulax circumspiciens.-Perkins, 1984: 330.

Type locality: Florida, Carysfort Reef, 48.3 $\mathrm{m}$ depth.

Type material: MCZ 662 (holotype).

20) **Myxicola sulcata Ehlers, 1912

Myxicola sulcata Ehlers, 1912: 29-30, Pl. 3,

Figs 5-12.- Hartman, 1966: 122, Pl. 40, Figs 4-7.

Type material:?

Type locality: Antarctic Ocean, Victoria Land, Ross Sea ("Discovery", Winter Quarters, No. 10 hole, 1.5 miles $\mathrm{S}$ of ship, on the edge of a bank bottom, small stones and organic debris to $237.7 \mathrm{~m}$ depth).
21) **Potamilla laciniosa Ehlers, 1905: 66-67, Pl. 9, Figs 7-10.

Type locality: New Zealand, Summer.

Type material: ZMH P-1335 (holotype).

Remarks: A junior synonym of Potamilla oligophthalmus Grube fide Johansson 1927: 151.

22) ***Potamilla perlonga Ehlers, 1908: 156.

Type locality: Indian Ocean, Off Nicobar, $296 \mathrm{~m}$ depth.

Type material: ?

Remarks: Subjective synonym of Potamilla paulina Grube (as Sabella) fide Johansson 1927: 146.

23) ***Potamethus spathiferus (Ehlers, 1887)

Potamis spathiferus Ehlers, 1887: 278-283,

Pl. 54, Figs 7-11, Pl. 55, Figs. 1-4.

Potamethus spathiferus. - Knight-Jones, 1983: 269, 271.

Type locality: Florida, off Sambos, $453.75 \mathrm{~m}$. Type material: MCZ 800 (holotype).

Remarks: Chamberlin (1919) introduced the name Potamethus in order to replace the genus $\mathrm{Po}^{-}$ tamis Ehlers since it was preoccupied in Lepidoptera.

24) **Sabella sulcata Ehlers, 1897: 65.

Type locality: Zanzibar.

Type material: ZMH V-4592.

Remarks: A junior synonym of Sabella porifera Grube fide Hartman 1959: 565.

DIAGNOSIS OF SUBFAMILIES IN SABELLIDAE

Fabriciinae Rioja, 1923

Usually small worms $(\sim 1 \mathrm{~mm}$, rarely $1 \mathrm{~cm})$; abdomen with 2-4 segments (Fig. 1A, 1C); 2-3 pairs of radioles (Fig. 1A); branchial skeleton absent; branchial hearts present (Fig. 1F, in arrows); branchial lobes separated.

\section{Sabellinae Latreille, 1825}

Worms of variable size ( 1 $\mathrm{mm}$ to $45 \mathrm{~cm})$; abdominal segments numerous (Fig. 1B) except for Terebrasabella (3 segments, Fig. 1D); numerous pairs of radioles (Figs. 1B, O-P, 2B) except for Terebrasabella (2 pairs, Fig. 1D); branchial skeleton with cells in single, two or more rows; branchial hearts absent; branchial lobes fused dorsally. 

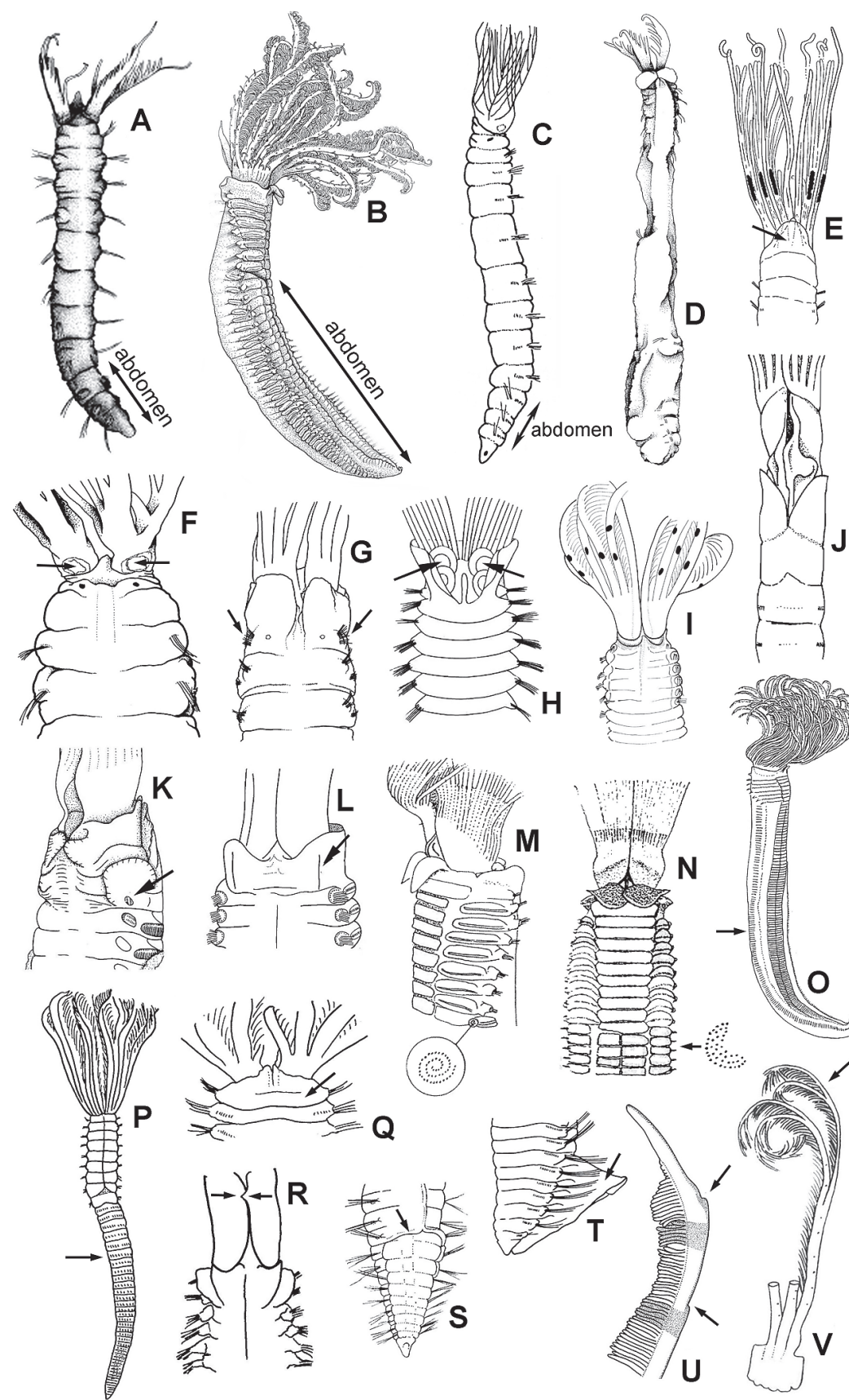

Fig. 1. Selected features of Sabellidae. A) Body, Novafabricia, B) body, Branchiomma, C) body, Fabricia, D) body, Terebrasabella, E) anterior peristomial ring, Desdemona, F) branchial hearts, Augeneriella, G) collar chaetiger, Panoumethus,

H) vascular coils, Fabrisabella, I) proximal compound eyes, Pseudopotamilla, J) collar, Potamethus, K) collar chaetiger,

Hypsicomus, L) collar chaetiger, Notaulax, M) spiral pattern, Sabella, N) C-shaped pattern, Bispira, O) body, Sabellastarte,

P) cinctures of abdominal uncini, Myxicola, Q) anterior peristomial ring, Amphiglena, R) S-shaped flanges in base of branchial crown, Stylomma, S) pre-pygidial depression with a distinct ridge, Euchone, T) pre-pygidial depression with lateral wings,

Euchone, U) pseudostylodes, Pseudobranchiomma, V) branched radiole, Schizobranchia. Figures modified from: A) Fitzhugh (1998), B) Rouse (2000), C) Fitzhugh (1989), D) Fitzhugh \& Rouse (1999), E) Banse (1957), F) Fitzhugh (1990a), G) Fitzhugh

(2002), H, S, V) Hartman (1969), I, L, U) Tovar-Hernández \& Salazar-Vallejo (2006), J) Knight-Jones (1983), K) Perkins

(1984), M-N) Knight-Jones \& Perkins (1998), O) Knight-Jones \& Mackie (2003), P-R) schematized, T) Banse (1972). 

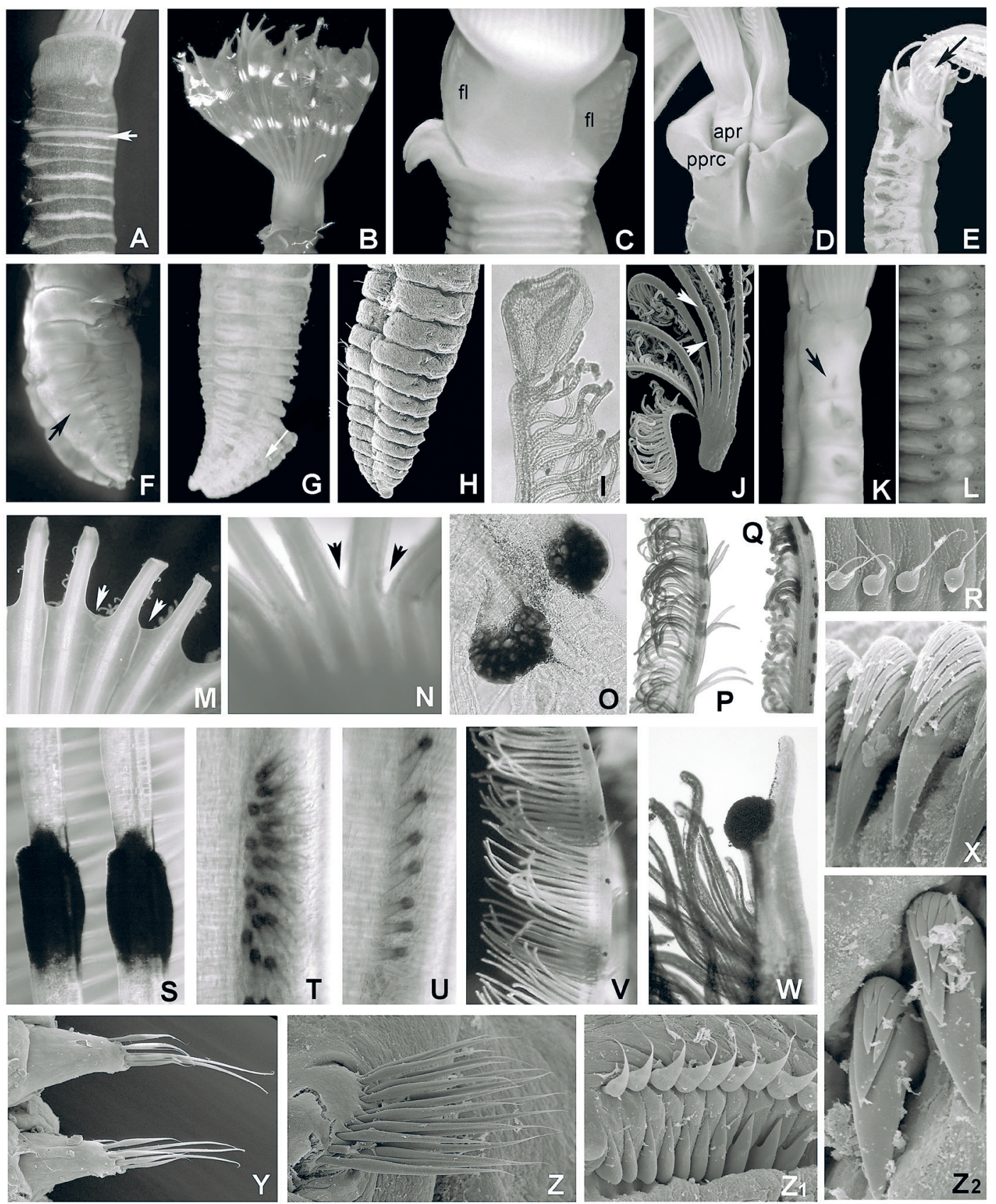

Fig. 2. Selected features of Sabellidae. A) Glandular ridge on chaetiger 2, Paradialychone, B) crown, Notaulax, C) basal flanges, Anamobaea, D) peristomium, Chone, E) abscission zone, Jasmineira, F-G) pre-pygidial depressions with lateral wings, Euchone, H) simple pre-pygidial depression, Dialychone, I) distal, radiolar flanges, Claviramus, J) proximal, compound eyes, Pseudopotamilla, K) collar chaetiger, Panousea, L) interamal eyespots, Branchiomma, M) high palmate membrane, Chone, N) low palmate membrane, Perkinsiana, O, S) paired compound eyes, Bispira, P) stylodes, Branchiomma, Q) ocular spots, Demonax, R) companion chaetae, Demonax, T) grouped eyes, Notaulax, U) row of eyes, Notaulax, V) compound eyes, Branchiomma, W) distal eyes, Megalomma, X) teeth of anterior abdominal uncini, Dialychone, Y) abdominal chaetiger, Branchiomma, Z) abdominal chaetiger, Megalomma, Z) location of companion chaetae, Megalomma, $\mathrm{Z}_{2}$ ) teeth of anterior abdominal uncini, Paradialychone. Pictures: B) Humberto Bahena, F, N) Beatriz Yáñez, S) Leslie Harris. Abreviations: apr anterior peristomial ring, fl flange, pprc posterior peristomial ring collar. 
KEY TO GENERA IN THE SUBFAMILY SABELLINAE

1 A whitish glandular ridge in the second chaetiger present (Fig. 2A, in arrow); thoracic uncini only acicular (Fig. 3D) ... 2

- A whitish glandular ridge in the second chaetiger absent; thoracic uncini not acicular (except for Terebrasabella) ... 15

2(1) Abdominal uncini form nearly complete cinctures around the body (Fig. 1P, in arrow); abdominal uncini with two or three teeth above main fang, without handles (Fig. 3E-F) ... Myxicola Renier in Meneghini 1847

- Abdominal uncini form short, discrete tori (Figs. 1O, 2G); abdominal uncini with numerous rows of teeth above the main fang ... 3

3(2) Palmate membrane absent ... 4

- Palmate membrane present (Fig. 2M-N) ... 8

4(3) Thoracic companion chaetae absent ... 5 $\left.2 Z_{1}\right)$

- Thoracic companion chaetae present (Fig.

5(4) Prominent, foliaceous flanges at the distal ends of the radioles (Fig. 2I) ... Claviramus Fitzhugh, 2002

-Foliaceous flanges at the distal end of radioles absent ... 6

6(5) Posterior peristomial ring collar absent; anterior margin of anterior peristomial ring developed ventrally as wide lobe (Fig. 1E, in arrow) ... Desdemona Banse, 1957

- Posterior peristomial ring collar present (Fig. 2D); abscission zone in the base of branchial crown present: refers to crowns where there is a distinct point immediately above the radiolar bases, where the radioles become detached from the branchial basis (Fig. 2e, in arrow) ... Jasmineira Langerhans, 1880

7(4) Bayonet chaetae present (Fig. 3G); collar chaetae fascicle (chaetiger 1) arranged as elongate row (Fig. 2K, in arrow); collar chaetae short, spinelike ... Panousea Rullier \& Amoureux, 1970

- Bayonet chaetae absent; collar chaetae fascicle (chaetiger 1) arranged as short, longitudinal row (Fig. 1G, in arrows) ... Panoumethus Fitzhugh, 2002

8(3) Posterior abdominal uncini similar to those in anterior abdomen ... 9

- Posterior abdominal uncini modified from those in anterior abdomen ... 13
9(8) Two pairs of distinct vascular coils visible dorsally below collar (Fig. 1H, in arrows) ... Fabrisabella Hartman, 1969

- Without vascular coils $\quad$... 10

10(9) Hood of thoracic uncini absent; posterior peristomial ring distinctly elongate, often extending anteriorly beyond collar (Fig. 1j) ... Potamethus Chamberlin, 1919

- Hood of thoracic uncini present (Fig. 3D, in arrow) ... 11

11(10) Abdominal uncini with handles well developed, rounded breast (Fig. 3J) ... Euchoneira Licciano, Giangrande \& Gambi, 2009

- Abdominal uncini without handles; breast hooked (Fig. 3H) or quadrangular (Fig. 3I) ... 12 12(11) Abdominal uncini with several regular, vertical rows of teeth, occupying at least threequarters of its length (rasp-shaped plates); hooked breast (Fig. 3H) ... Amphicorina Claparède, 1864

- Abdominal uncini with a main fang surmounted by a few rows of teeth, occupying one-quarter of its length; quadrangular breast (Fig. 3I) ... Chone Krøyer, 1856

13(8) Ventral margin of collar incised; prepygidial depression with lateral wings (Figs 1T, $2 \mathrm{~F}-\mathrm{G}$, in arrows) or with a distinct girdle marking the anterior and lateral edges of the depression (Fig. 1S, in arrow) ... Euchone Malmgren, 1866

- Ventral margin of collar entire (Fig. 2A); simple pre-pygidial depression (Fig. $2 \mathrm{H}$ ) $\quad \ldots 14$ 14(12) Uncini from anterior abdomen with a series of nearly uniform size (Fig. 2X) .... Dialychone Claparède, 1870

- Uncini from anterior abdomen with a large tooth above main fang, followed by a series of smaller teeth (Fig. $2 \mathrm{Z}_{2}$ ) ... Paradialychone TovarHernández, 2008

15(1) Two or three types of thoracic uncini: acicular (Fig. 3K), avicular (Fig. 3L) and palmate (Fig. $3 \mathrm{~A}-\mathrm{C}$ ); three abdominal chaetigers (Fig. 1D); living in burrows of the shells of marine gastropods, coral rubble or inside spirorbid tubes ... Terebrasabella Fitzhugh \& Rouse, 1999

- Only one type of thoracic uncini; abdomen with numerous segments (Fig. 1B) _.. 16

16(15) Abdominal neuropodia as erect, conical lobes, elevated (Fig. 2Y) _.. 17

- Abdominal neuropodia as low, transverse ridges (Fig. 2Z) ... 23 
17(16) Companion chaetae present (Fig. $\left.2 Z_{1}\right)$

- Companion chaetae absent $\quad \ldots 218$

18(17) Base of branchial crown with a long base and S-shaped dorsal basal flanges (Fig. 1R); superior abdominal chaetae absent; radioles with stalked terminal compound eyes (absent in one species) ... Stylomma Knight-Jones, 1997

- Base of branchial crown with a short base without flanges; superior abdominal chaetae present; stalked terminal compound eyes absent ... 19

19(18) Companion chaetae hook-shaped (Fig. 3O-P); thoracic and abdominal uncini without handle; living in calcareous tubes ... Glomerula Brünnich Nielsen, 1931 (synonym of Calcisabella Perkins, 1991)

- Companion chaetae with distal ends as distinctly assymetrical membranes (Fig. 3Q-R); thoracic and abdominal uncini with handles (Fig. 3J, U); living in sandy tubes ... 20

20(19) Abdominal neurochaetae arranged in a tight spiral pattern (Fig. 1M); radiolar eyes absent ... Sabella Linnaeus, 1767

- Abdominal chaetae arranged in C-shaped pattern containing further arcs or random infilling (Fig. 1N); radioles with paired (rarely single) composite eyes (Fig. 2S) distributed along radiolar length (absent in four species) ... Bispira Krøyer, 1856

21(17) Radioles with stylodes (Fig. 2P) or pseudostylodes (flanges) (Fig. 1U) _.. 22

- Radioles without stylodes ... Sabellastarte Krøyer, 1856

22(21) Radioles with pseudostylodes (Fig. $1 \mathrm{U})$; compound radiolar eyes can be present (only in 5 of the 13 species), whereas paired or arranged in irregular assemblages ... Pseudobranchiomma Jones, 1962

- Radioles with stylodes (Fig. 2p); compound radiolar eyes always present, paired (Fig. 2O, V) ... Branchiomma Kölliker, 1858

23(16) Abdomen with paleate neurochaetae, with mucro (Fig. 3V-W, Y) ... 24

- Abdomen without paleate neurochaetae ... 26

24(23) Base of the branchial crown with erect, dorsal and ventral flanges (Fig. 2C) ... Anamobaea Krøyer, 1856

- Base of the branchial crown without flanges
25(24) Chaetiger 1 with chaetae (collar chaetae) arranged in a transversal row (elongate fascicle) (Figs 1L); lensed ocelli in a continuous row (Fig. 2U) on lateral margins distal to palmate membrane, or scattered, or organized into various groups (Fig. 2T) ... Notaulax Tauber, 1879

- Chaetiger 1 with chaetae (collar chaetae) arranged in a fascicle (bundle similar to other superior thoracic notochaetae) (Fig. 1K); lensed ocelli scattered on lateral margins distal to palmate membrane ... Hypsicomus Grube, 1870

26(23) Inferior thoracic notochaetae composed of paleate (Fig. 3Y) or broadly hooded chaetae (Fig. 3X) ... 27

- Inferior thoracic notochaetae composed of paleate chaetae only (Fig. 3Y) ... 29

27(26) At least dorsalmost radioles with compound eyes located on inner margins, near distal end (Fig. 2W) ... Megalomma Johansson, 1925

- Radioles without compound eyes located on inner margins, near distal end; radioles with spots (Fig. 2Q) or unpaired, simple eyespots along entire lengths of outer radiole margins (Fig. 2V) ... 28

28(27) Companion chaetae with rounded denticulate heads and long, drop shaped, slightly asymmetrical membranes (Fig. 2Z); interramal, simple ocelli in all chaetigers ... Sabellomma Nogueira, Fitzhugh \& Rossi, 2010

- Companion chaetae with membranes pointed, perpendicular to slender shaft (Fig. 2R); without interramal, simple ocelli ... Parasabella Bush, 1905 (reintroduced as a replacement name for Demonax Kinberg, 1867)

29(26) With unpaired radiolar compound eyes present on outer margins; limited to proximal region of most radioles (Figs 1I, 2J) _.. 30

- Without compound radiolar eyes ... 32

30(29) Radioles, at least of large specimens, appear spiraled; very numerous (more than 20 pairs); sometimes dichotomously branched (Fig. 1V) ... Eudistylia Bush, 1905

- Radioles not spiraled, less than 20 pairs ... 31

31(30) Radioles with numerous dichotomous branches (Fig. 1V) ... Schizobranchia Bush, 1905

- Radioles not branched ... Pseudopotamilla Bush, 1905

32(29) Radioles united by a low palmate membrane (Fig. 2N) ... Potamilla Malmgren, 1866

- Radioles free to their bases _... 33 
33(32) Thoracic uncini replaced by thick spines (Fig. 3S) on last few chaetigers (in chaetigers 5-7) ... Potaspina Hartman, 1969

- Thoracic uncini not replaced by spines (all thoracic neurochaetae are uncini of similar shape)

34(33) Last five abdominal chaetigers with uncini replaced by large, thick, falcate spines (Fig. 3T) ... Sabellonga Hartman, 1969

- All abdominal uncini of similar shape ... 35

35(34) Posterior peristomial ring collar absent (Fig. 1Q, in arrow) ... Amphiglena Claparède, 1864
- Posterior peristomial ring collar present ... 36

36(35) Thoracic uncini with handles of variable length, 2-5 times the length of main fang (Fig. 3U).... Perkinsiana Knight-Jones, 1983

- Thoracic uncini without handles (Fig. 3M) or with very reduced handles (Fig. 3Z) ... 37

37(36) Thoracic and abdominal uncini with a very short handle (Fig. 3Z-Z $_{1}$... Aracia Nogueira, Fitzhugh \& Rossi, 2010 (substitute name for Kirkia Nogueira, López \& Rossi, 2004)

- Thoracic and abdominal uncini without handles (Fig. 3M-N) ... Laonome Malmgren, 1866.

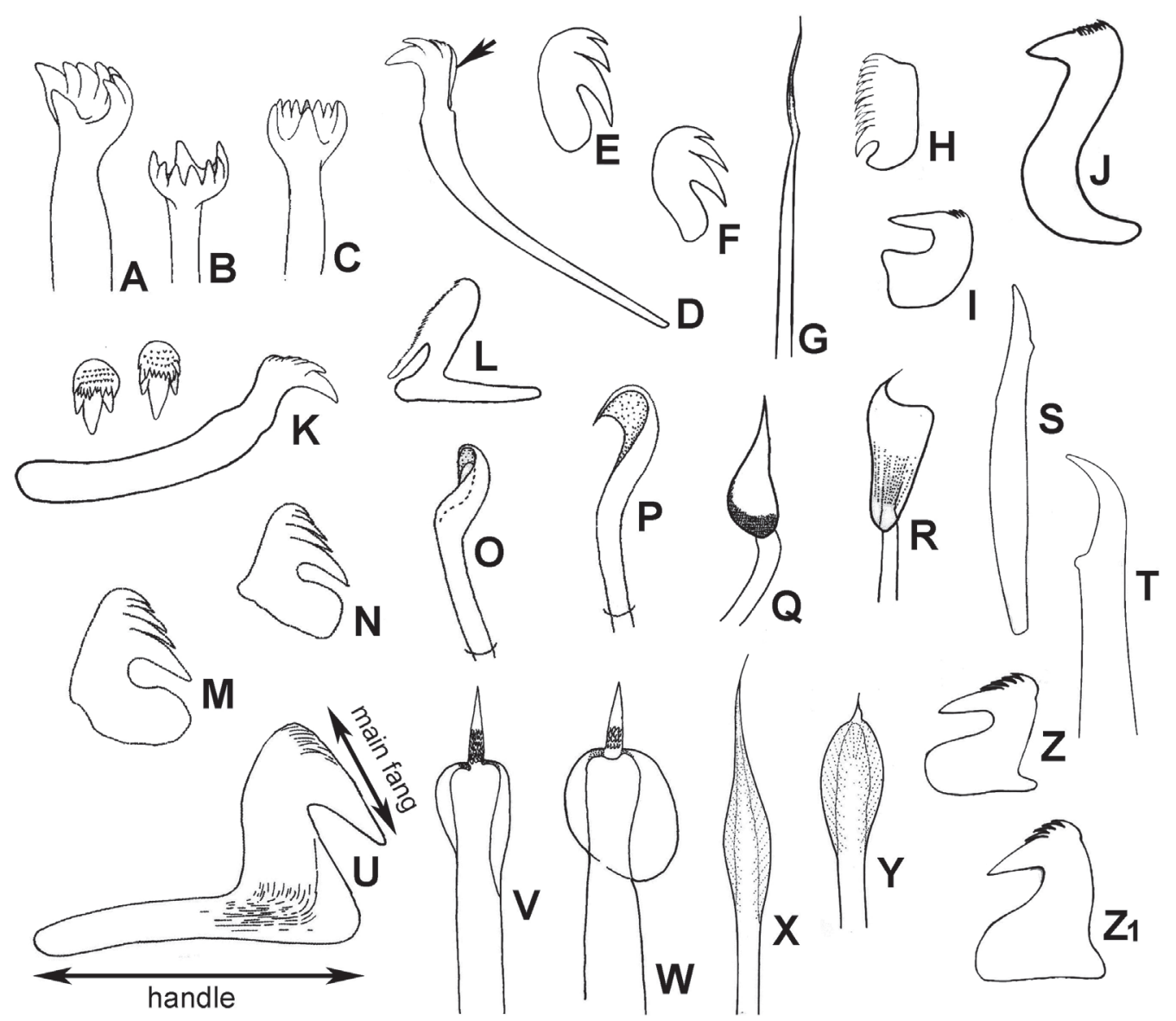

Fig. 3. Selected chaetae and uncini of Sabellidae. A-C) Palmate hooks, D, K) thoracic, acicular uncini, E-F, $\mathrm{H}-\mathrm{J}, \mathrm{N}, \mathrm{Z}_{1}$ ) abdominal uncini, G) bayonet chaeta, O-P) companion chaetae hooked-shaped, Q-R) companion chaetae teardrop-shaped, S-T) aciculae, U, Z) thoracic avicular uncini, V-W) abdominal paleate chaetae, X) broadly hooded chaeta, Y) paleate chaeta. Figures modified from: A-C) Jones (1974), E-F, M-N) Fitzhugh (2002), G-I, Q-R, X-Y) Fitzhugh (1989), J) Banse (1972), K-L) Fitzhugh \& Rouse (1999), O-P) Perkins, 1991,

S-T) Hartman (1969), V-W) Tovar-Hernández \& Salazar-Vallejo (2006), Z-Z ) Nogueira et al. (2004). 


\section{ACKNOWLEDGEMENTS}

I am grateful to Dr. Américo Montiel San Martín (Instituto de la Patagonia, Universidad de Magallanes) for their kind invitation to write this contribution. Kathrin Philipps-Bussau and Petra Wagner (Zoologisches Museum, Universität Hamburg) provided valuable curatorial information. Clara Ramírez (ICML-UNAM), João Gil (CEAB-SCIC) and Beatriz Yáñez-Rivera (ICML-UNAM) were helpful locating and sending me literature.

\section{LITERATURE CITED}

Annenkova, N. 1934. Kurze Übersicht der Polychaeten der Litoralzone der Bering-Insel (Kommador-Inseln) nebst Beschreibung neuer Arten. Zoologischer Anzeiger 106(12): 322-331.

Annenkova, N. 1937. Polychaete fauna of the northern part of the Japan Sea. Issledovaniya fauny morei, Zoologicheskii Institut Akademii Nauk USSR 23: 139-216.

Augener, H. 1922a. Litorale Polychaeten von Juan Fernandez. In: C. Skottsberg (Ed.). The Natural History of Juan Fernandez and Eastern Island, pp. 161-218.

Augener, H. 1922b. Über Litorale Polychaeten von Westindien. Gesellschaft der Naturforschender Freunde zu Berlin, 38-63.

Augener, H. 1926. Papers from Dr. Th. Mortensen's Pacific Expedition 1914-16. XXXIV. Polychaeta III. Polychaeten von Neuseeland. II Sedentaria. Videnskabelige Meddelelser fra Dansk naturhistorisk Forening i Köbenhaun 81: 157-294.

Augener, H. 1932. Zoologische Ergebnisse der Reisen von Dr. Kohl-Larsen nach den Subantarktischen Inseln bei Neuseeland und nach Südgeorgien. 8. Polychaeten. Senckenbergiana, Frankfurt am Main 14(3): 95-117.

Banse, K. 1957. Die Gattungen Oriopsis, Desdemona und Augeneriella (Sabellidae, Polychaeta). Videnskabelige Meddelelser fra Dansk naturhistorisk Forening i Köbenhaun 119: 67-105.

Banse, K. 1972. Redescriptions of some species of Chone Kröyer and Euchone Malmgren, and three new species (Sabellidae, Polychaeta).
Fishery Bulletin, Fish and Wildlife Service, United States Department of Interior 70(2): 459-495.

Banse, K. 1979. Sabellidae (Polychaeta) principally from the northeast Pacific Ocean. Journal of the Fisheries Research Board of Canada 36(8): 869-882.

Benham, W.B. 1927. Polychaeta. British Antarctic 'Terra Nova' Expedition Natural History Reports, Zoology 7(2): 47-182.

Brünnich Nielsen, K. 1931. Serpulidae from the Senonian and Danian deposits of Denmark. Meddelelser fra Dansk Geologisk Forening, Københaun 8: 71-113.

Bush, K.J. 1905 Tubicolous annelids of the tribes Sabellides and Serpulides from the Pacific Ocean. Harriman Alaska Expedition 12: 169-346.

Cantone, G. 1972. Richerche sulla fauna e sulla zoogeographia della Sicilia 54. Pseudofabricia aberrans n. gen. n. sp., un anellide policheti di incerta sede. Bollettino delle sedute dell'Accademia Gioenia di Scienze Naturali in Catania 11(3-4): 1-7.

Capa, M. 2007. Taxonomic revision and phylogenetic relationships of apomorphic sabellids (Polychaeta) from Australia. Invertebrate Systematics 21: 537-567.

Capa, M., P. Hutchings, M.T. Aguado \& N.J. Bott 2010. Phylogeny of Sabellidae (Annelida) and relationships with other taxa inferred from morphology and multiple genes. Cladistics 26: $1-21$.

Caullery, M. \& F. Mesnil 1896. Note sur deux serpuliens nouveaux (Oriopsis metchnikowi n.g., n.sp. et Josephella marenzelleria n.g., n. sp.). Zoologischer Anzeiger 10: 482-486.

Chamberlin, R.V. 1919. The annelida polychaeta of the Albatross tropical Pacific expedition, 1891-1905. Memoirs of the Museum of Comparative Zoology at Harvard College 48: 1-514.

Claparède, E. 1864. Glanures zootomiques parmi les annélides de Port-Vendres (Pyrénées Orientales). Mémoires de la Société de physique et d'histoire naturelle de Genève 17(2): 463-600.

Claparède, E. 1870. Les Annélides Chétopodes du Golfe de Naples. Seconde partie. Annélides 
sédentaires. Mémoires de la Société de physique et d'histoire naturelle de Genève 20(1): 1-225.

Day, J.H. 1955. The Polychaeta of South Africa. Part 3. Sedentary species from Cape shores and estuaries. Journal of the Linnean Society of London 42(287): 407-452.

Day, J.H. 1961. The Polychaeta Fauna of South Africa, 6. Sedentary species dredged off Cape coast with a few new records from the shore. Journal of the Linnean Society of London 44: 463-560.

Day, J.H. 1967. A Monograph on the Polychaeta of Southern Africa. British Museum of Natural History 656: 38-878.

de Blainville, H. 1828. Dictionnaire des Sciences naturelles, dans lequel on traite méthodiquement des differens êtres de la nature, considérés soit en eux-mêmes, d'après l'état actuel de nos connaisance, soit relativement a l'utilité qu'en peuvent retirer la médicine, l'agriculture, le commerce et les arts. Suivi d'une biographie des plus célèbres naturalistes. FG Levrault, Strasbourg \& Paris 57: 1-536.

de Quatrefages, A. 1866. Histoire naturelle des Annelés marins et d'eau douce. Annélides et Géphyriens. Librarie Encyclopédique de Roret, Paris, Vol. 1: 588 pp.

de Saint-Joseph, B. 1894. Les Annélides polychètes des côtes de Dinard. Troisième Partie. Annales des Sciences Naturelles, Paris 17: 1-395.

Ehlers, E. 1871. Ueber die aud fer von Heuglin Waldurgschen Expedition nach Spitzbergen gesammelten Würmer. Sitzungesberichte der Phys. Med. Soc, Erlangen, Spitzber 77-86.

Ehlers, E. 1887. Report on the Annelids. FloridaAnneliden. Reports on the Result of Dredging, under the direction of Pourtalès, during the years 1868-1870, and of Alexander Agassiz, in the Gulf of Mexico (1877-78), and in the Caribbean Sea (1878-79), in the U.S. Coast Survey steamer "Blake", Lieut. Com. C.D. Sigsbee, U.S.N., and Commander J.R. Bartlett, U.S.N., Commanding. Memoirs of the Museum of Comparative Zoology, Harvard University 15: 1-335.

Ehlers, E. 1897. Polychaeten. Hamburger Magalhaensische Sammelreise, Friederichsen, Hamburg 148 pp.
Ehlers, E. 1901a. Die Polychaeten des magellanischen und chilenischen Strandes: Ein faunisticher Versuch. Fetschrift zur Feier des 150 jährigen Bestehens der Königlichen Gesellschaft der Wissenschaften zu Göttingen. Weidmannsche Buchhandlung, Berlin.

Ehlers, E. 1901b. Fauna Chilensis. Die Anneliden der Sammlung Plate. Zoologisches Jahrbücher Jena, (Suppl 5): 251-272.

Ehlers, E. 1904. Neuseeländische Anneliden. Abhandlung der Koeniglichen Gesellschaft der Wisseschaften zu Göttingen, MathematikPhysik, Klasse 3: 1-80.

Ehlers, E. 1905. Anneliden der Sammlung Schauinsland. Ergebnisse einer Reise nach dem Pacific. Schauinsland 1896/97. Zoologische Jahrbücher, Abteilung für Systematik, Geographie und Biologie der Tiere 22(3): 281-302.

Ehlers, E. 1907. Neuseeländische Anneliden, 2. Abhandlungen der Königlichen Gesellschaft der Wissenschaften zu Göttingen, Mathematisch-Physikalische Klasse, Neue Folge 5: 1-31.

Ehlers, E. 1908. Die Bodensaessigen Anneliden aus den Sammlungen der deutschen Tiefsee-Expedition. In: C. Chun (Ed.). Wissenschaftliche Ergebnisse der deutschen Tiefsee-Expedition auf dem Dampfer 'Valdivia' 1898-1899, Lief, Vol. 16, pp. 1-168.

Ehlers, E. 1912. Polychaeta. British Museum (Natural History), National Antarctic Expedition, Natural History 6: 1-32.

Ehlers, E. 1913. Die Polychaeten-Sammlungen der deutschen Südpolar- Expedition 19011903. Deutsche Südpolar Expedition 13(5): 397-598.

Ehlers, E. 1920. Polychaeten von Java und Amboina. Ein Beitrag zur Kenntnis der malaiischen Strandfauna. Abhandlungen der koeniglichen Gesellschaft der Wissenschaften zu Goettingen 10(7): 1-73.

Ehrenberg, C.G. 1836. Mehrere kleine Thiere der Nordsee, aus Helgoland und einige aus dem Cattegat bei Gothenburg. Mitteilungen der Gesellschaft naturforschende Freunde zu Berlin for 1-5.

Fauchald, K. 1977. Polychaetes from intertidal areas in Panama, with a review of previous shallow water records. Smithsonian Contribution to Zoology 221: 1-81. 
Fauchald, K. 2007. World Register of Polychaeta, available online at http://www.marinespecies.org.

Fauvel, P. 1916. Annélides polychètes des Iles Falkland receuillies par M. Rupert Vallentin Esq. (1902-1910). Archives de zoologie expérimentale et générale 55: 417-482.

Fauvel, P. 1927. Polychètes Sedentaires \& Addenda aux Polychètes Errantes. Faune de France 16: $1-494$.

Fauvel, P. 1936. Polychètes d'Expédition antarctique Belgique. pp 1-46. Resultats du Voyage de la Belgica en 1897-1899, sour le commandement de A. de Gerlache de Gomery, Anvers.

Fitzhugh, K. 1989. A systematic revision of the Sabellidae-Caobangiidae-Sabellongidae complex (Annelida: Polychaeta). Bulletin of the American Museum of Natural History 192: 104.

Fitzhugh, K. 1990a. A revision o the Fabriciin genus Augeneriella Banse, 1957 (Polychaeta: Sabellidae). Journal of Natural History 24: 195-218.

Fitzhugh, K. 1990b. Fabricinuda, a new genus of Fabriciinae (Polychaeta: Sabellidae). Proceedings of the Biological Society of Washington 103: 161-178.

Fitzhugh, K. 1990c. Two new genera of the subfamily Fabriciinae (Polychaeta: Sabellidae). American Museum Novitates 2967: 1-19.

Fitzhugh, K. 1992. On the systematic position of Monroika africana (Monro) (Polychaeta: Sabellidae: Fabriciinae) and a description of a new fabriciin genus and species from Australia. Proceedings of the Biological Society of Washington 105: 116-131.

Fitzhugh, K. 1998. New fan worm genera and species (Polychaeta, Sabellidae, Fabriciinae) from the Western Pacific, and cladistic relationships among genera. Zoologica Scripta 27: 209-245.

Fitzhugh, K. 2001. A new deep-water genus and species of Fabriciinae fanworm (Polychaeta: Sabellidae) from Antarctica. Natural History Museum of Los Angeles County, Contributions Science 491: 1-8.

Fitzhugh, K. 2002. Fan worm polychaetes (Sabellidae: Sabellinae) collected during the Thai-Danish Bioshelf Project. Phuket Marine Biological Center, Special Publications 24: 353-424.
Fitzhugh, K. \& G.W. Rouse 1999. A remarkable new genus and species of fan worm (Polychaeta: Sabellidae: Sabellinae) associated with marine gastropods. Invertebrate Biology 118: 357-390.

Friedrich, H. 1939. Polychaeten-Studien V-X. Zur Kenntnis einiger wenig bekannter oder neuer Polychaeten aus der westlichen Ostsee. Kieler Meeresforsch 3(2): 362-373.

Giangrande, A. 1992. The genus Chone (Polychaeta, Sabellidae) in the Mediterranean Sea with description of $C$. longiseta n. sp. Bolletino di Zoologia 59: 517-529.

Giangrande, A. 1994. The genus Demonax (Polychaeta, Sabellidae) in the Mediterranean Sea, with description of $D$. tommasi n. sp. Bollettino di Zoologia 61: 229-233.

Giangrande, A. \& M.C. Gambi 1997. The genus Perkinsiana (Polychaeta, Sabellidae) from Antarctica, with descriptions of the new species $P$. milae and $P$. borsibrunoi. Zoologica Scripta 26(3): 267-278.

Giangrande, A., P. Montanaro \& A. Castelli 1999. On some Amphicorina (Polychaeta: Sabellidae) species from the Mediterranean coast, with the description of $A$. grahamensis. Italian Journal of Zoology 66: 195-203.

Glasby, C.J. \& G.B. Read 1998. A chronological review of polychaete taxonomy in New Zealand. Journal of the Royal Society of New Zealand 28: 347-374.

Gravier, C. 1906. Contribution a l'étude des annélides polychetes de la Mer Rouge. Nouvelle Archives du Muséum d'Histoire Naturelle, sér. 4, 8: 123-236.

Gravier, C. 1908. Contribution a l'étude des annélides polychetes de la Mer Rouge (suite). Nouvelle Archives du Muséum d'Histoire Naturelle, sér. 4, 10: 67-168.

Grube, A.E. 1870. Bemerkungen über Anneliden des Pariser Museum. Archiv für Naturgeschichte, Berlin 36(1): 281-352.

Hartman, O. 1951. Fabriciinae (feather-duster polychaetous annelids) in the Pacific. Pacific Science 5: 379-391.

Hartman, O. 1953. Non-pelagic Polychaeta of the Swedish Antarctic Expedition 1901-1903. In: N.H. Odhner (Ed). Further Zoological Result on the Swedish Antarctic Expedition 1901-1903, pp 1-83. 
Hartman, O. 1959. Catalogue of the Polychaetous Annelids of the World. Part 2. Occasional Papers of the Allan Hancock Foundation 23(2): 355-628.

Hartman, O. 1961. Polychaetous annelids from California. Allan Hancock Pacific Expeditions 25: $1-226$.

Hartman, O. 1965. Catalogue of the Polychaetous Annelids of the World. Supplement 1960-5 and Index. Occasional Papers of the Allan Hancock Foundation 23(Supp): 1-197.

Hartman, O. 1966. Polychaeta Myzostomidae and Sedentaria of Antarctica. Antarctic Research Series 7: 1-158.

Hartman, O. 1967. Polychaetous annelids collected by the USNS Eltanin and Staten Island cruises, chiefly from Antarctic Seas. Allan Hancock Monographs in Marine Biology 2: 1-387.

Hartman, O. 1969. Atlas of the Sedentariate Polychaetous Annelids from California. Allan Hancock Foundation, USC, Los Angeles, 812.

Hartman, O. 1978. Polychaeta from the Weddell Sea Quadrant, Antarctica. Antarctic Research Series 26(4): 125-222.

Hartmann-Schröder, G. 1962. Die Polychaeten des Eulitorals. Zur Kenntnis des Eulitorals der chilenischen Pazifikküste und der argentinischen Küste Südpatagoniens unter besonderer Berücksichtigung der Polychaeten und Ostracoden. Mitteilungen aus dem Hamburgischen Zoologischen Museum und Institut 60 (Suppl): 57-167.

Hartmann-Schröder, G. 1965. Die Polychaeten des Sublitorals. Zur Kentnnis des Sublitorals der chilenischen Küste unter besonderer Berücksichtigung der Polychaeten und Ostracoden. (Mit Bemerkungen über den Einfluss sauerstoffarmer strömungen auf die Besiedlung von marinen Sedimenten). Mitteilungen aus dem Hamburgischen Zoologischen Museum und Institut 62 (Suppl): 59-305.

Hartmann-Schröder, G. 1971. Annelida, Borstenwürmer, Polychaeta. In: M. Dahl \& P. Fritz (Eds). Tierwelt Deutschlands und der angrenzenden Meeresteile nach ihren Maermalen und nach ihrer Lebensweise. Gustav Fischer Verlag, Jena, pp. 1-594.

Hartmann-Schröder, G. 1983. Die Polychaeten der 15, 36 und 76. Reise von FFS 'Walter Herwig'
Pseudobranchiomma and Sabellastarte. In: C. Dauvin, L. Laubier \& D.J. Reish (Eds) Actes de la 4éme Conférence Internationale des Polychètes. Memoires du Muséum Nationale d'Histoire Naturelle 162: 191-198.

Hartmann-Schröder, G. 1996. Annelida, Borstenwürmer, Polychaeta. Die Tierwelt Deutschlands 58 vol. Second edition, Fischer, Jena, 645 pp.

Hartmann-Schröder, G. \& P. Rosenfeldt 1991. Die Polychaeten der 'Walther Herwig'-Reisen 68/1 nach Elephant Island (Antarkis) 1985. Teil 2: Acrocirridae bis Sabellidae. Mitteilungen aus dem Hamburgischen zoologischen Museum und Institut 88: 73-96.

International Commission of Zoological Nomenclature 1999. International code of zoological nomenclature. Fourth edition. London: The International Trust for Zoological Nomenclature.

Johansson, H.P. 1922. On some new tubicolous annelids from Japan, the Bonin Islands and the Antarctic. Arkiv för Zoologi 15(2): 1-11. Johansson, H.P. 1925. Bemerkungen über die Kinberg'schen Arten der Familien Hermellidae und Sabellidae. Arkiv för Zoologi 18A(7): 1-28.

Johansson, H.P. 1927 Beiträge zur Kenntnis der Polychaeten-Familien Hermellidae, Sabellidae und Serpulidae. Zoologiska Bidrag fran Uppsala 11: 1-184.

Johnson, H.P. 1901. The Polychaeta of the Puget Sound region. Proceedings of the Boston Society for Natural History 29: 381-437.

Jones, M.L. 1962. On some polychaetous annelids from Jamaica, the West Indies. Bulletin of the American Museum of Natural History 124: $169-212$.

Kinberg, J.G.H. 1867. Annulata nova. Öfversigt af Königlich Vetenskapsakademiens förhandlingar, Stockholm 23(9): 337-357.

Kinberg, J.G.H. 1910. Andra Delen. Zoologi 3. Annulater. Kongliga Svenska Fregatten Eugenies Resa omkring jorden under befal af C.A. Virgin aren 1851-1853. Vetenskapliga Iakttagelser pa Konung Oscar den Forstes befallningutgifna af K. Svenska Vetenskapsakademien. Almquist and Wicksells Boktryckeri A/B, Uppsala \& Stockholm.

Knight-Jones, P. 1983. Contributions to the taxonomy of Sabellidae (Polychaeta). Zoological Journal of the Linnean Society 79: 245-295. 
Knight-Jones, P. 1994. Two new species of Branchiomma (Sabellidae) with redescriptions of closely related species and comments on Pseudobranchiomma and Sabellastarte. In: C. Dauvin, L. Laubier \& D.J. Reish (Eds) Actes de la 4éme Conférence Internationale des Polychètes. Mémoires du Muséum Nationale d'Histoire Naturelle 162: 191-198.

Knight-Jones, P. 1997. Two new species of $\mathrm{Me}^{-}$ galomma (Sabellidae) from Sinai and New Zealand with redescriptions of some types and a new genus. Bulletin of Marine Science 60: 313-323.

Knight-Jones, P. \& N. Bowden 1984. Incubation and scissiparity in Sabellidae (Polychaeta). Journal of Marine Biological Association United Kingdom 64: 809-818.

Knight-Jones, P. \& A.S.Y. Mackie 2003. A revision of Sabellastarte (Polychaeta: Sabellidae). Journal of Natural History 37: 2269-2301.

Knight-Jones, P. \& T.H. Perkins 1998. A revision of Sabella, Bispira and Stylomma (Polychaeta: Sabellidae). Zoological Journal of Linnean Society 123: 385-467.

Knight-Jones, P. \& A.J.M. Walker 1985. Two new species of Demonax (Sabellidae: Polychaeta) from Liverpool Bay. Journal of Natural History 19: 605-612.

Kohn, A.J. \& M.C. Lloyd 1973. Marine polychaete annelids of Easter Island. Internationale Revue der gesammten Hydrobiologie 58(5): 691-712.

Kölliker, H. 1859. Über Kopfkiemer mit Augen an den Kiemen (Branchiomma dalyelli). Zeitschrift für wissenschaftliche Zoologie 9: 356-541.

Krøyer, H. 1856. Bidrag til Kundskab af Sabellerne. K Danske Videnskabers Selskabs Forhandlinger 1856: 1-36.

Kupriyanova, E.K \& G.W. Rouse 2008. Yet another example of paraphyly in Annelida: Molecular evidence that Sabellidae contains Serpulidae. Molecular Phylogenetics and Evolution 46: 1174-1181.

Langerhans, P. 1880. Die Wurmfauna von Madeira, II. Zeitschrift für wissenschaftliche Zoologie 33: 271-316.

Latreille, M. 1825. Familles Naturelles du Règne Animal exposées succinctement et dans un ordre analytique avec l'indication de leurs genres etc. JB Baillière, Paris, 570 pp.
Leidy, J. 1859. Manayunkia speciosa. Journal of the Academy of Natural Sciences of Philadelphia 10: 90.

Levinsen, M.R. 1883. Systematisk-geograhiskOversigt over de nordiske Annulata, Gephyrea, Chaetognathi og Balanoglossi. Videnskabelige Meddelelser fra den Naturhistoriske Forening i Kjobenhaun 1882: 160-250.

Licciano M., A. Giangrande \& M.C. Gambi 2009. A new genus of Sabellidae (Annelida, Polychaeta) from Antarctica, with discussion of relationships among plesiomorphic genera within Sabellinae. Zootaxa 2226: 28-42.

Linnaeus, C. 1767. Systema naturae per regna tria naturae, secundum classes, ordines, genera, species, cum characteribus, differentiis, synonymis, locis. Stockholm.

Malmgren, A.J. 1866. Nordiska Hafs-Annulater. Öfversigt af Kongl Vetenskaps-Akademiens Förhandlingar, Stockholm 22(5): 355-410.

McIntosh, W.C. 1923. A monograph of the British marine annelids. Polychaeta, Sabellidae to Serpulidae. With additions to the British marine Polychaeta during the publication of the monograph. Royal Society of London 4: 251-538.

Meneghini, G. 1847. Osservazioni postume di Zoologia Adriatica. Instituto Neneto, Venezia, 57-66.

Monro, C.C.A. 1930. Polychaete worms. Discovery Reports, Cambridge 2: 1-222.

Monro, C.C.A. 1936. Polychaete worms II. Discovery Reports, Cambridge 12: 59-193.

Montiel, A., C. Ríos, E. Mutschke \& N. Rozbaczylo 2004. Poliquetos de fiordos y canales adyacentes al Campo de Hielo Patagónico Sur, Chile (Annelida: Polychaeta). Ciencia y Tecnología del Mar 27(1): 49-67.

Moore, J.P. 1904. New Polychaeta from California. Proceedings of the Academy of Natural Sciences of Philadelphia 56: 484-503.

Moreno, R., P. Neill \& N. Rozbaczylo 2006. Native and non-indigenous boring polychaetes in Chile: a threat to native and commercial mollusc species. Revista Chilena de Historia Natural 79: 263-278.

Murray, A. \& G.W. Rouse 2007. Two new species of Terebrasabella (Annelida: Sabellidae: Sabellinae) from Australia. Zootaxa 1434: 51-68. 
Nogueira, J.M.D. \& A.C.Z. Amaral 2000. Amphicorina schlenzae, a small sabellid (Polychaeta, Sabellidae) associated with a stony coral on the coast of São Paulo State, Brazil. Bulletin of Marine Science 67(1): 617-624.

Nogueira, J.M.M., K. Fitzhugh \& M.C.S. Rossi 2010. A new genus and new species of fan worms (Polychaeta: Sabellidae) from Atlantic and Pacific Oceans -the formal treatment of taxon names as explanatory hypotheses. Zootaxa 2603: 1-52.

Nogueira, J.M.M., E. López \& M.C.S. Rossi 2004. Kirkia heterobranchiata, a new genus and species of extratubular brooding sabellid (Polychaeta: Sabellidae) from São Paulo, Brazil. Journal of Marine Biological Association United Kingdom 84: 701-710.

Perkins, T.H. 1984. Revision of Demonax Kinberg, Hypsicomus Grube, and Notaulax Tauber, with a review of Megalomma Johansson from Florida (Polychaeta: Sabellidae). Proceedings of the Biological Society of Washington 97(2): 285-368.

Perkins, T.H. 1991. Calcisabella piloseta, a new genus and species of Sabellinae (Polychaeta: Sabellidae). Bulletin of Marine Science 48: 261-267.

Pixell, H.L.M. 1913. Polychaeta of the families Serpulidae and Sabellidae, collected by the Scottish National Antarctic Expedition. Transactions of the Royal Society of Edinburgh 49(2): 347-358.

Rioja, E. 1917. Datos para le conocimiento de la fauna de Anélidos Poliquetos del Cantábrico. Trabajos del Museo Nacional de Ciencias Naturales 29: 1-111.

Rioja, E. 1923. Estudio sistemático de las especies Ibéricas del suborden Sabelliformia. Trabajos del Museo Nacional de Ciencias Naturales, Serie Zoológica 48: 1-144.

Ríos, C., W.E. Arntz, D. Gerdes, E. Mutschke \& A. Montiel 2007. Spatial and temporal variability of the benthic assemblages associated to the holdfasts of the kelp Macrocystis pyrifera in the Straits of Magellan, Chile. Polar Biology 31: 89-100.

Rouse, G.W. 2000. Sabellida. In: P.L. Beesley, G.J.B. Ross \& C.J. Glasby (Eds) Polychaetes \& Allies: The Southern Synthesis. Fauna of
Australia, 4A. Polychaeta, Myzostomida, Pogonophora, Echiura, Sipuncula. CSIRO Publishing, Melbourne, pp. 172-173.

Rouse, G.W. 1994. New species of Oriopsis Caullery and Mesnil from Florida, Belize, and Aldabra Atoll (Seychelles), and a new species of Amphiglena Claparède from Seychelles (Polychaeta: Sabellidae: Sabellinae). Bulletin of Marine Science 54: 180-202.

Rouse, G.W. 1996. New Fabriciola and Manayunkia species (Fabriciinae: Sabellidae: Polychaeta) from Papua New Guinea. Journal of Natural History 30: 1761-1778.

Rozbaczylo, N. 1985. Los anélidos poliquetos de Chile: Indice sinonímico y distribución geográfica de especies. Facultad de Ciencias Biológicas, Pontificia Universidad Católica de Chile, Monografías Biológicas 3: 1-284.

Rullier, F. 1954. Essai de revision du genre Fabricia (Annélides Polychetes). Bulletin de la Société Zoologique de France 79: 14-29.

Rullier, F. \& L. Amoureux 1970. Nouvelle contribution a l'etude de la faune des annélides polychètes du Maroc. Bulletin de la Société des Sciences Naturelles et Physiques du Maroc 1969: 109-142.

Sars, M. 1851 Beretning om en i Sommeren 1849 foretagen zoologisk Reise i Lofoten og Finmarken. Nyt Magazin for Naturvidenskaberne, Christiania 2(2): 121-211.

Sars, M. 1862. Foredrag om de ved Norges Kyster forekommende Arter af den Linnéiske Annelideslæegt Sabella. Forhandlinger $i$ Videnskabsselskabet i Kristiania 1862, 116-133.

Savigny, J.S. 1822. Système des Annélides, principalement de celles des côtes de l'Égypte et de la Syrie, offrant les caractères tant distinctifs que naturelles des ordres, familles et genres, avec la description des espèces. Description de l'Égypte, Vol. 3, Part 1. Paris: Imprimerie Impériale, $128 \mathrm{pp}$.

Schmarda, L.K. 1861. Neue wirbellose Thiere beobachtet und gesammelt auf einer Reise um die Erde 1853 bis 1857. Neue Turbellarien, Rotatorien und Anneliden, Vol. I, Pt. II. Whilhelm Engelmann, Leipzig, 164 pp.

Tauber, P. 1879. Annulata Danica. En Kritisk Revision af de i Danmark Fundne Annulata 
Chaetognatha, Gephyrea, Balanoglossi, Discophoreae, Oligochaeta, Gymnocopa og Polychaeta. Reitzel, Kobenhavn 1-143.

Thomson, J. 1860. Essai d'une classification de la famille des cérambycides et matériaux pour servir à une monographie de cette famille, Paris $396 \mathrm{pp}$.

Tovar-Hernández, M.A. 2007. On some species of Chone Krøyer, 1856 (Polychaeta: Sabellidae) from world-wide localities. Zootaxa 1518: 31-68.

Tovar-Hernández, M.A. 2008. Phylogeny of Chone Kröyer, 1856 (Poychaeta: Sabellidae) and related genera. Journal of Natural History 42(33-34): 2193-2226.

Tovar-Hernández, M.A. \& L.H. Harris 2010. Parasabella Bush, 1905, replacement name for the polychaete genus Demonax Kinberg, 1867 (Annelida: Polychaeta: Sabellidae). Zookeys 60: 13-19.

Tovar-Hernández, M.A. \& P. Knight-Jones 2006. Species of Branchiomma (Polychaeta: Sabellidae) from the Caribbean Sea and Pacific coast of Panama. Zootaxa 1189: 1-37.

Tovar-Hernández, M.A. \& S.I. Salazar-Vallejo 2006. Sabellids (Polychaeta: Sabellidae) from the Grand Caribbean. Zoological Studies 45(1): 24-66.
Tovar-Hernández, M.A. \& S.I. Salazar-Vallejo 2008. Caruncle in Megalomma Johansson, 1925 (Polychaeta: Sabellidae) and the description of a new species from the Eastern Tropical Pacific. Journal of Natural History 42(29-30): 1951-1973.

Tovar-Hernández, M.A. \& T. Sosa-Rodríguez 2006. Redescription of Chone infundibuliformis Krøyer, 1856 (Polychaeta: Sabellidae) and histology of the branchial crown appendages, collar and glandular ridge. Zootaxa 1115: 31-59.

Tovar-Hernández, M.A., N. Méndez \& T.F. Villalobos-Guerrero 2009. Fouling polychaetes worms from the southern Gulf of California: Sabellidae and Serpulidae. Systematics and Biodiversity 7: 1-18.

Treadwell, A.L. 1924. Dasychonopsis arenosa, a new species of polychaetous annelids from Porto Rico. American Museum Novitates 107: $1-2$.

Webster, H.E. 1884. Annelida from Bermuda, collected by G. Brown Goode. Bulletin of the United States National Museum 25: 305-327.

Zenkevitsch, L.A. 1925. Polychaeta of Beluschja Bay, Novaya Zemblja. Trudy Wissenschaftliche Meeresuntersuchungen Institut Moscow 1: 1-12. 
